\title{
Morphological and Optical Characterization of High Density Au/PAA Nanoarrays
}

\author{
Mohamed Shaban \\ Nanophotonics and Applications (NPA) Laboratory, Department of Physics, Faculty of Science, Beni-Suef University, \\ Salah Salm Street, Beni-Suef 62514, Egypt
}

Correspondence should be addressed to Mohamed Shaban; mssfadel@aucegypt.edu

Received 17 February 2016; Accepted 6 April 2016

Academic Editor: Davide Ferri

Copyright (C) 2016 Mohamed Shaban. This is an open access article distributed under the Creative Commons Attribution License, which permits unrestricted use, distribution, and reproduction in any medium, provided the original work is properly cited.

Hexagonal nanoarrays of Au nanorods and nanopillar were deposited on nanoporous anodic alumina (PAA) membranes utilizing dc electrodeposition. The surface morphologies and optical properties were characterized by using field emission-scanning electron microscopy (FE-SEM) and UV-Vis spectrophotometer, respectively. The optical reflectance spectra of the as-prepared, pore widened, and 2D-Au nanorods-coated PAA membranes were studied in detail. The effects of the angle of incident, pore widening time, and electrodeposition time on the characteristic peaks positions and intensities of the fabricated nanoarrays were addressed. As the angle of incident increased, the interference peaks and transverse surface resonance are shifted to longer wavelengths, but the longitudinal surface plasmon resonance is shifted to shorter wavelengths. Also, the reflected intensities are decreased linearly for the as-prepared sample and decreased exponentially for Au/PAA samples. Using the modified Kubelka-Munk radiative transfer model, the energy gap is increased from 2.83 to $3.06 \mathrm{eV}$ and the refractive index is decreased from 1.84 to 1.36 for the as-prepared and $70 \mathrm{~min}$ pore widened PAA membranes, respectively. Based on the advantages of the fabrication approach and the enhanced and controlled properties, this generation of Au/PAA arrays can be used as efficient building blocks for nanoelectronics and nanophotonic devices.

\section{Introduction}

In recent years, porous materials have attracted much interest due to their wide range of applications [1-3]. Among them, nanoporous anodic alumina (PAA) has been widely used as membrane in the fabrication of various nanostructures $[3,4]$. Two-step anodization was extensively used to fabricate PAA membranes because of the cheap equipment, simple technology, and high controllability of the process [5]. The applications of the PAA membranes can be extended by depositing metal nanostructures inside their nanopores or on their top surfaces $[4,6]$. So the conducting, mechanical, and optical properties of PAA membranes could be improved $[1,6]$. Different nanotubes, nanorods, nanoparticles, and nanowires have been successfully deposited inside the pores of PAA membranes [7-9]. However, uniform and low-price deposition of metal nanostructures inside the pores of PAA has gained much attention due to their extensive use as substrates for sensors based on surface-enhanced Raman scattering (SERS), laser induced fluorescence (LIF), and electrochemical measurements [3, 10-12].

On the other hand, the optical properties of the PAA membranes have been extensively addressed by many authors [12-15]. Because PAA membranes showed bright colors with very tiny saturation, then, colored nanostructures always have to be sealed with their nanopores to increase and modify the color saturation [16, 17]. Also, the optical properties of Au nanoarrays have been extensively studied [6, 18-20] to identify their surface plasmon resonances interactions. These surface plasmon interactions can be used to enhance, guide, and modify the optical fields of novel nanoarrays such as photonic crystals [21], sensors based on SERS [11, 22], laser induced fluorescence, and near-field microscopy [3]. In particular, $\mathrm{Au}$ nanoarrays showed the features of both nanostructured $\mathrm{Au}$ that exhibit localized surface plasmon resonances (LSPRs) and planar Au films that show propagating surface plasmon resonances (PSPRs) $[6,19,23]$. Also, the internal coupling between Au nanoarrays leads to energy 
shifts and splitting of degenerate modes, an effect that is prominent for regular nanoarrays [24].

In our previous studies, different metallic nanostructures have been loaded on the outer surface of PAA membranes such as $\mathrm{Au}, \mathrm{Cr}, \mathrm{Pt}$, and $\mathrm{Sn}[12,19,25,26]$. Also, the effects of different parameters such as Au-particles size, pore diameter, PAA thickness, the state of polarization, and angle of the incident on the optical properties were addressed [4, 19]. These studies showed surface plasmon enhancement of PAA interference. Recently, Shaban studied the effect of PAA pore thickness and state of polarization of the incident light on the surface plasmon modes of hexagonal arrays of $\mathrm{Au}$ nanoparticles grown around the active dots on the top surface of PAA membrane by r.f. magnetron sputtering [4]. In addition, different methods have been used to deposit Au nanorod arrays inside the pores of PAA. Among them, electrodeposition is one of the most promising methods because it is a simple, low-cost, and high-throughput technique [27]. Previously, we used combined cathodic polarization (CP) and pore widening (PW) method to remove the hemispherical barrier layer (10$30 \mathrm{~nm}$ ), which prevent the homogenous electrodeposition, between PAA pores and Al substrate [28]. Then Au nanorods of $100 \%$ filling factor were deposited inside the pores of PAA membranes without bottom barrier layers [6]. We showed only the effect of the aspect ratio and angle of the incident on the surface plasmon resonance of Au nanorods under the effect of the polarized light [6]. However, other factors can affect the optical properties of these nanostructures including the deposition time, the morphology of nanorods, pore diameters of the PAA membrane, and the angle of incident of the unpolarized light. All of these factors make it necessary to have experimental data that can correlate the parameters of the deposited Au nanoarrays inside PAA pores with the optical properties. Here, we studied the effect of combined (CP and PW) method and electrodeposition time on the PAA and Au/PAA morphologies and optical properties under illumination of the unpolarized light incident at different angles.

\section{Sample Fabrication and Characterization}

Two-step anodization combined with CP and PW processes was utilized to fabricate PAA membranes with uniform pore diameters $[6,28]$. The 1st anodization process was performed in $0.3 \mathrm{M}$ oxalic acid at $40 \mathrm{~V}$ and $9^{\circ} \mathrm{C}$ for $3 \mathrm{~h}$. After the removal of the nonuniform nanoporous alumina layer, the second anodization was performed at the same conditions for $10 \mathrm{~min}$ and followed by the barrier-thinning process. The barrier layer was thinned by a successive drop of the DC voltage from 40 to $15 \mathrm{~V}$ at a rate of $0.1 \mathrm{~V} / \mathrm{s}$ and then maintained at $15 \mathrm{~V}$ for $5 \mathrm{~min}$. The pore widening was carried out for 60 or $70 \mathrm{~min}$ after $10 \mathrm{~min}$ cathodic polarization $[6,28]$. The $\mathrm{Au}$ nanorods were deposited inside the PAA membranes from a cyanide-free Au electroplating solution (ECF-33B manufactured by N.E. Chemcat Corporation, Japan) using dc electrodeposition of current $5 \mathrm{~mA}$ at $57^{\circ} \mathrm{C}$ for different lengths of time [6].
Field emission-scanning electron microscope (FE-SEM, JSM-7500F/JEOL) was used to study the morphological and structural properties of the fabricated nanoarrays. Optical reflectance in the spectral range from 350 to $1000 \mathrm{~nm}$ were measured using UV/VIS/NIR 3700 double beam Shimadzu spectrophotometer at RT.

\section{Results and Discussion}

3.1. Morphological Study. FE-SEM characterized the surface properties of the as-prepared, posttreated, and Au electrodeposited PAA membranes. Figure 1(a) illustrates top view and cross-sectional view FE-SEM images of the as-prepared PAA membrane. Hexagonal aligned nanopores were formed in PAA membrane. The pore diameter is $\sim 26.5 \pm 3 \mathrm{~nm}$, the interpore distance is $\sim 100 \mathrm{~nm}$, and the pore density is $\sim 1.13 \times 10^{10} \mathrm{~cm}^{-2}$. The inset of the top and cross-sectional FE-SEM images clearly shows the existence of some flocks like $\mathrm{Al}_{2} \mathrm{O}_{3}$ inside the nanopores, which affect the regular circular shape of the pores. From the cross-sectional image, the thickness of the PAA layer is $667 \mathrm{~nm}$, and then the rate of growth is $66.7 \mathrm{~nm} / \mathrm{min}$. The inset of the cross-sectional image illustrates the existence of the hemispherical barrier layer of thickness $<60 \mathrm{~nm}$.

Figures 1(b) and 1(c) show FE-SEM images of PAA membranes posttreated with $10 \mathrm{~min} \mathrm{CP}$ followed by (b) $60 \mathrm{~min}$ and (c) $70 \mathrm{~min}$ PW process. As displayed in the top view and cross-sectional view images, by increasing the pore widening time, the regularity of the pores increased, the number of defects or cracks decreased, and homogeneity of the hexagonal arrangement increased. Also, the pore diameter is increased to $71.4 \pm 2.1$ and $78.5 \pm 1.2 \mathrm{~nm}$ for $60 \mathrm{~min}$ and $70 \mathrm{~min}$ PW, respectively. Then, the rate of pore widening of the pores was found to be $0.75 \mathrm{~nm} / \mathrm{min}$. From the crosssectional image of Figure 1(b), the thickness of the barrier layer is decreased to $<15 \mathrm{~nm}$ after $60 \mathrm{~min}$ PW. Moreover, the cross-sectional image, Figure 1(c), indicates the complete removal of the barrier layer.

Figure 2 shows FE-SEM images of PAA of pore diameters 78.5, 71.4, and $71.4 \mathrm{~nm}$, respectively, electrodeposited with $\mathrm{Au}$ at $5 \mathrm{~mA}$ and $57^{\circ} \mathrm{C}$ for (a) $3 \mathrm{~min}$, (b) $4 \mathrm{~min}$, and (c) $6 \mathrm{~min}$. High density and well aligned hexagonal Au nanorod arrays grew vertically inside the PAA pores over a large area, as shown in Figure 2(a). The filling factor of the pores is $100 \%$. The average rode diameter of the deposited Au nanoarray is $\sim 85.7 \mathrm{~nm}$, which is greater than the diameter of the starting PAA pores; this implies that the pores are enlarged during the electrodeposition process. This may be ascribed to the etching effect of the Au electroplating solution and temperature [29]. Also, the array height is almost equal to the pore height $(667 \mathrm{~nm})$, and the aspect ratio is $\mathrm{AR}=7.8$. At some pores, the pore walls are cracked, and Au nanorods are coalesced, as indicated by the arrows.

To check the effect of the deposition time on the morphology of the produced nanorod array, we used two PAA membranes of smaller pore diameter $(71.4 \mathrm{~nm})$ fabricated by decreasing the PW time to $60 \mathrm{~min}$ as working electrode during the electrodeposition. By increasing the deposition time from $4 \mathrm{~min}$ to $6 \mathrm{~min}$, the morphology of the nanorods 

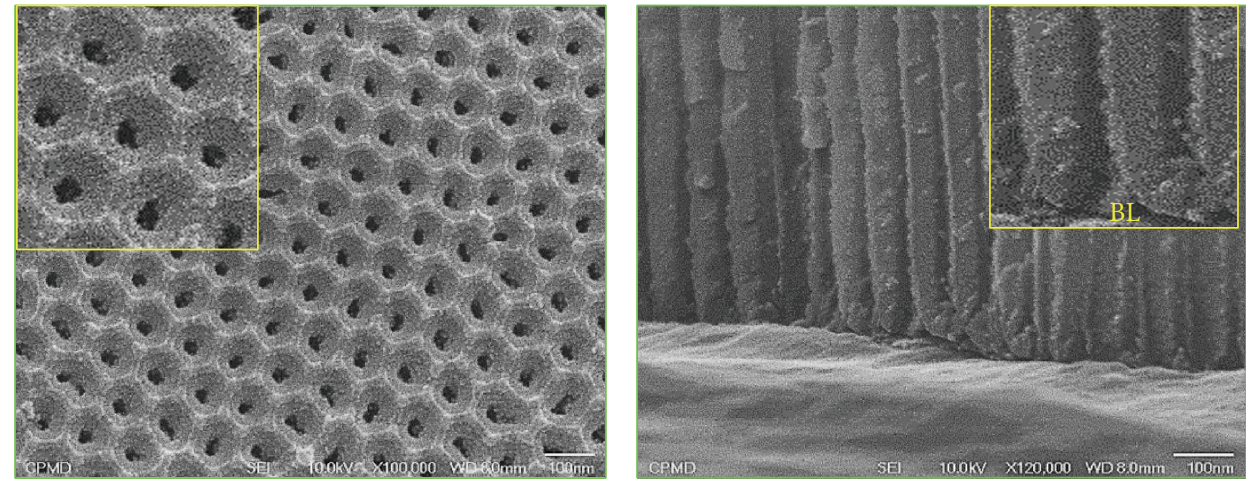

(a)
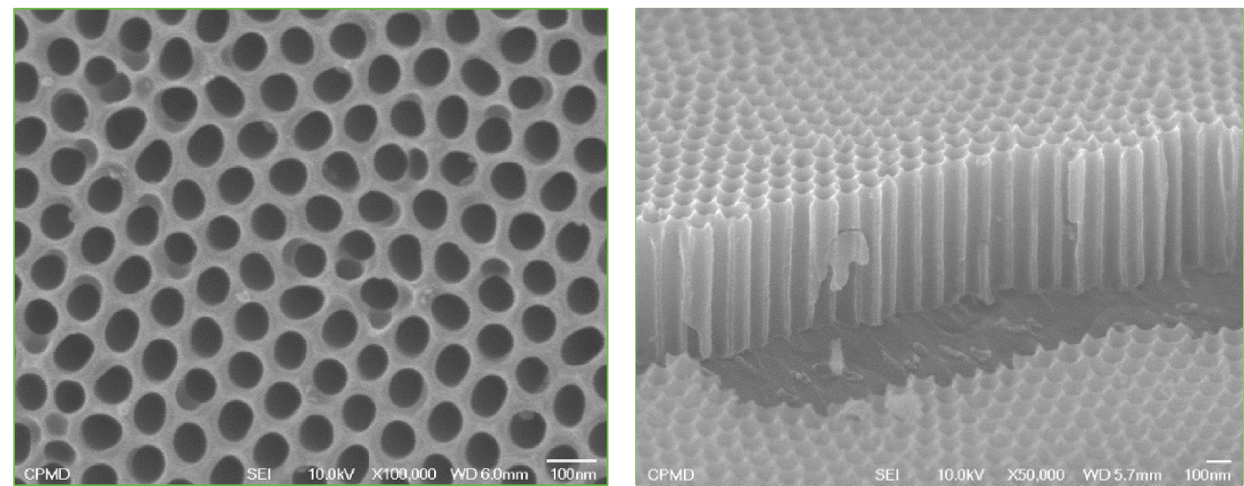

(b)
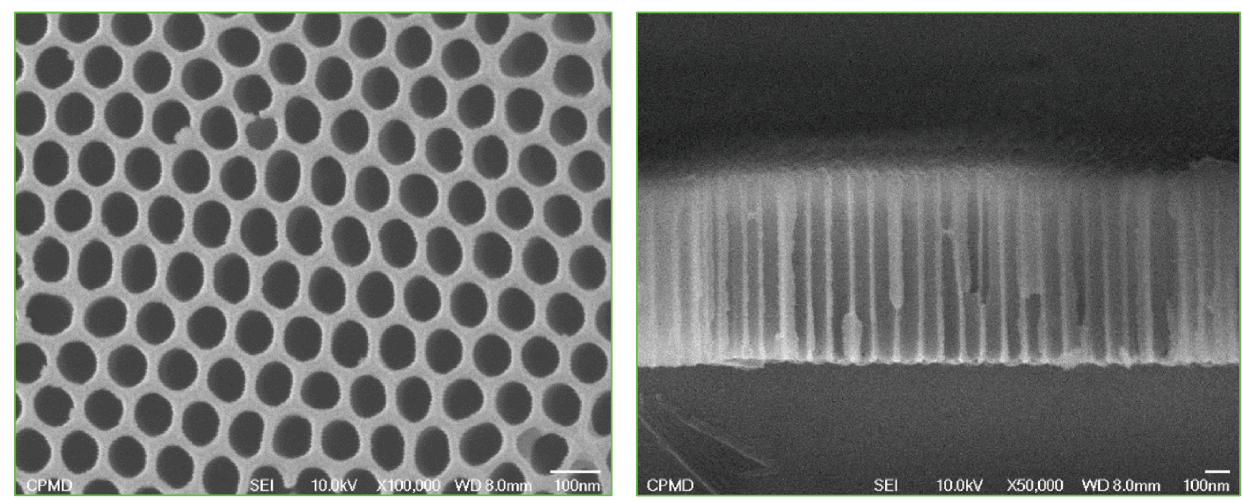

(c)

FIGURE 1: Top view, left column, and cross-sectional view, right column, FE-SEM images of PAA membranes (a) as-prepared after $t_{2 \text { nd }}=$ $10 \mathrm{~min}$, (b) after posttreatment of $t_{\mathrm{CP}}=10 \mathrm{~min}$ and $t_{\mathrm{PW}}=60 \mathrm{~min}$, and (c) after posttreatment of $t_{\mathrm{CP}}=10 \mathrm{~min}$ and $t_{\mathrm{PW}}=70 \mathrm{~min}$.

is tuned from cylindrical shape to pillar shape as shown in Figures 2(b) and 2(c). The height of the deposited nanorods was greater than the height of the PAA pores. As shown from this figure, the homogeneity and hexagonal arrangement of the Au nanorods are improved in addition to the controllability of height and diameter. The average diameter of the deposited nanorods is approximately $78.4 \mathrm{~nm}$. The improved structural properties of the deposited $\mathrm{Au}$ nanorods can be attributed to the uniform removal of the hemispherical barrier layer, which caused improved adhesion to the substrate and better height and diameter control and uniformity of nanorods.
3.2. Optical Properties of PAA Membranes and Au/PAA Composites. Figure 3(a) shows reflectance spectra of as-prepared PAA membrane measured at different angles of the incident in increments of $1 \mathrm{~nm}$. These spectra were normalized with respect to a reflection from an aluminum mirror. The average reflectivity of PAA is changed from $75 \%$ to $67 \%$ as the angle of incident increased. This means that the scattering coefficient and hence the effect of scattering are minuscule for this PAA membrane for $\lambda \geq 400 \mathrm{~nm}$. Well-resolved interference dips and peaks are observed in the reflection spectra, which implies the high quality of the fabricated PAA membranes. These peaks and dips are originated from 


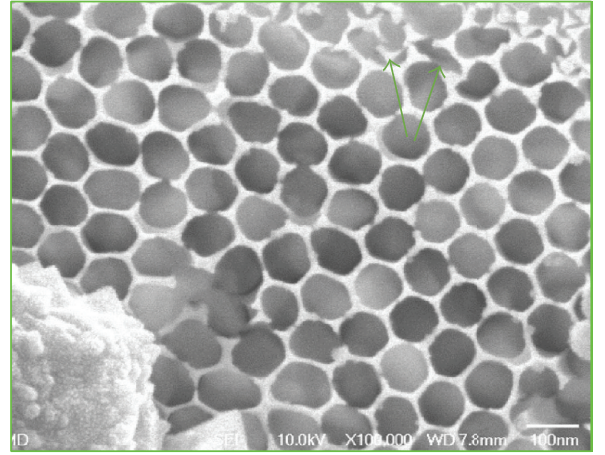

(a)

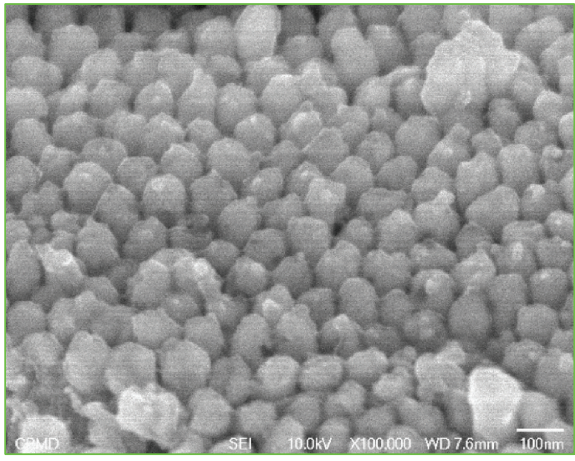

(b)

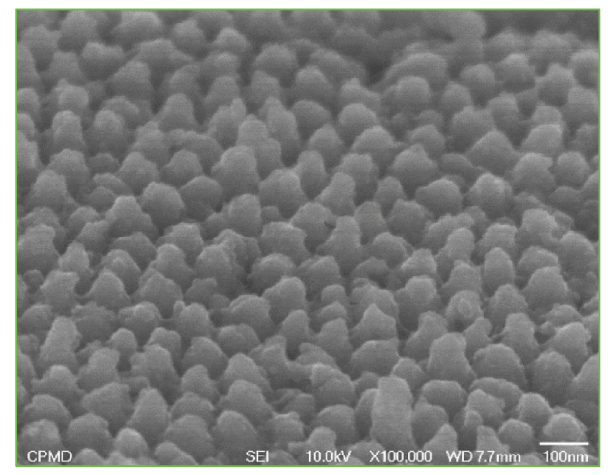

(c)

Figure 2: Top view FE-SEM images of (a) PAA membrane pore widened for 70 min and electrodeposited with Au at $5 \mathrm{~mA}$ and $57^{\circ} \mathrm{C}$ for 3 min and PAA membranes pore widened for $60 \mathrm{~min}$ and electrodeposited at the same conditions for (b) $4 \mathrm{~min}$ and (c) $6 \mathrm{~min}$.

the constructive and destructive interferences between the reflected waves from $\mathrm{Al} / \mathrm{PAA}$ and $\mathrm{PAA} /$ air interfaces. The width of the peaks increases and contrast decreases as the wavelength is increased. By increasing the angle of incidence, the contrast of the interference fringes decreases. Increasing the angle of incident shifts the peaks in the reflectance spectra linearly to longer wavelengths as shown in Figure 3(b), which is consistent with Bragg's law. As shown in the inset values of Figure 3(b), the separation between the peaks, $\Delta \lambda$, is increased as the angle of incident and wavelength increased. Additionally, the reflectance \% of the peaks decreases linearly for all the peaks as shown in Figure 3(c). As the incident angle changed, the effective pore diameter and effective dielectric constant for that light are also changed according to the modified Maxwell-Garnet effective medium approximation [19]. The absorption band below $400 \mathrm{~nm}$ is associated with the reduction in the absorption coefficient. The interference peak around $450 \mathrm{~nm}$ is much stronger than the others. This may be ascribed to the blue emission band of PAA which can be attributed to mixed emission from $\mathrm{F}$ and $\mathrm{F}^{+}$centers as discussed by Nasir et al. [30].

Using Bragg's law and interference fringes in the reflectance spectra of Figure 3(a), the effective refractive index, $n_{\text {eff }}$, can be calculated using [19]

$$
n_{\mathrm{eff}}^{2}=\frac{\lambda_{1}{ }^{2} \lambda_{2}{ }^{2}}{\left[4 d^{2}\left(\lambda_{1}-\lambda_{2}\right)^{2}\right]}+\sin ^{2} \theta
$$

where $\lambda_{1}$ and $\lambda_{2}$ are the wavelengths of two adjacent maxima or minima, $\theta$ is the angle of incident, and $d$ is the thickness of PAA. The calculated average value of $n_{\text {eff }}$ was found to be $1.84 \pm 0.15$.

The energy of the band gap can be calculated from the diffused reflectance spectrum, Figure 3(a), by applying the modified Kubelka-Munk radiative transfer model [31]. The absorbance can be calculated using $A=R_{\max }-R$, where $R_{\max }$ is the maximum value of reflectance for wavelengths longer than that of the dip in the diffuse reflectance and $R$ is the reflectance. The inset of Figure 3(a) shows the calculated absorbance spectrum for the PAA membrane. The band gap wavelength and hence energy gap can be obtained by extrapolating the long-wavelength edge of the peak in absorbance to the zero line of absorbance. The value of the calculated band gap energy of the as-prepared membrane was found to be $2.83 \pm 0.019 \mathrm{eV}$.

Figure $3(\mathrm{~d})$ shows reflectance spectra measured at a different angle of incident for PAA membrane after posttreatment of $10 \mathrm{~min}$ CP and $70 \mathrm{~min}$ PW. The dominant features in these spectra were similar to those of Figure 3(a), but the fringes are wider, weaker, and more shifted to longer wavelengths. Also, the behavior of the peak position versus the angle of incident is almost exponential as shown in Figure 3(e). Moreover, the variation of the peak reflectance with the angle of incident is presented in Figure 3(f), which shows a linear decrease in peaks I and III and nonlinear 

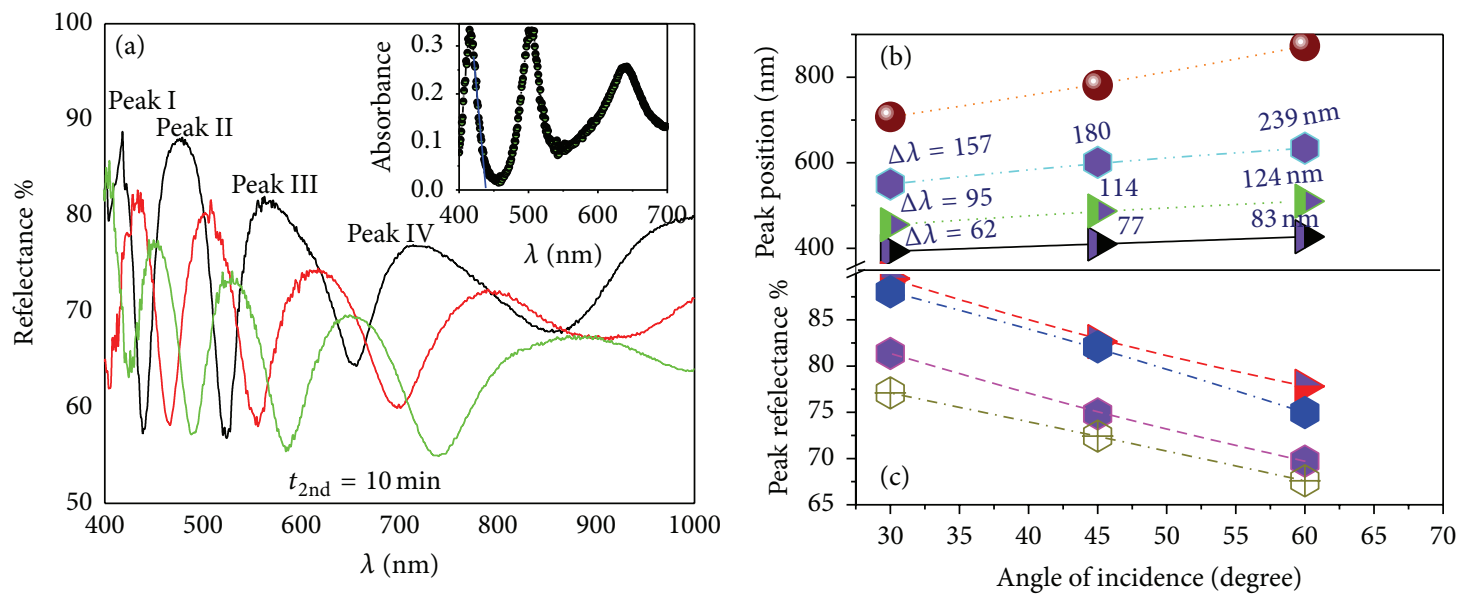

$$
\begin{aligned}
\theta & =30^{\circ} \\
\theta & =45^{\circ} \\
\theta & =60^{\circ}
\end{aligned}
$$
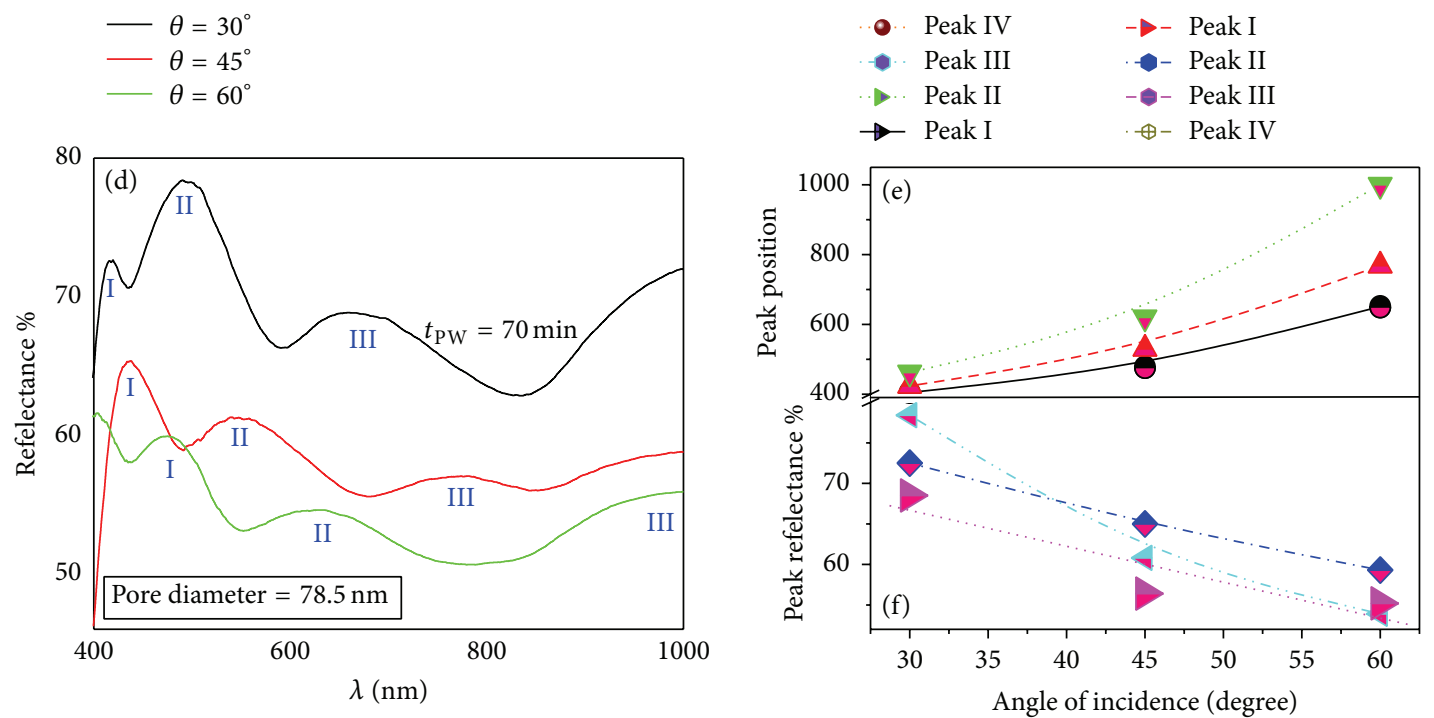

$\begin{aligned}-\theta & =30^{\circ} \\ \theta & =45^{\circ} \\ \theta & =60^{\circ}\end{aligned}$
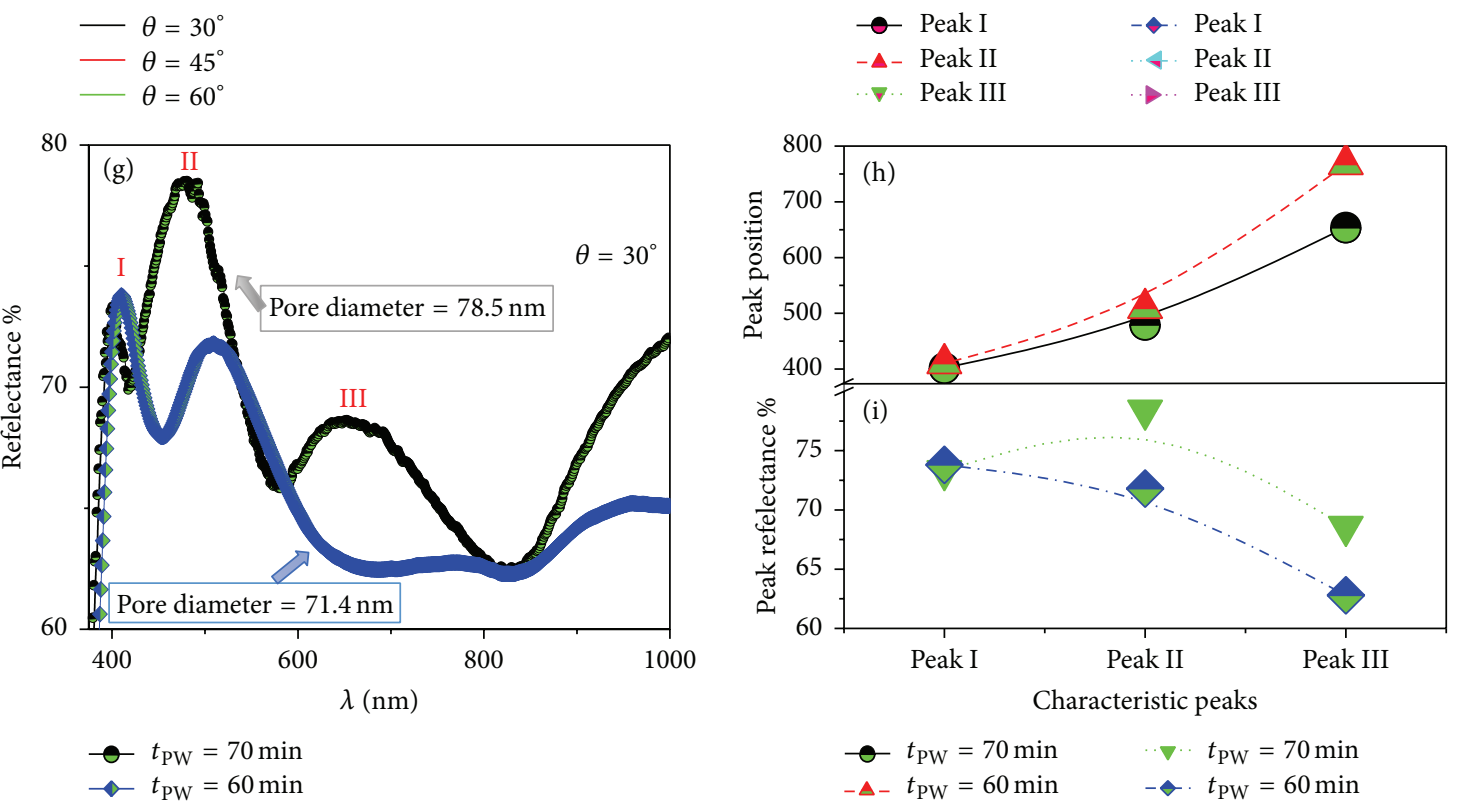

FIGURE 3: Reflection spectra of PAA membranes (a) as-prepared after 10 min second anodization and (d) after posttreatment of 70 min pore widening measured at different angles of incident. The variation of peak position (b, e) and peak reflectance $\%$ (c, f) as a function of the angle of incident. The effect of $60 \mathrm{~min}$ and $70 \mathrm{~min}$ pore widening time on (g) reflection spectra of PAA membrane, (h) peak position, and (i) peak reflectance $\%$ at $30^{\circ}$ angle of incidence. 

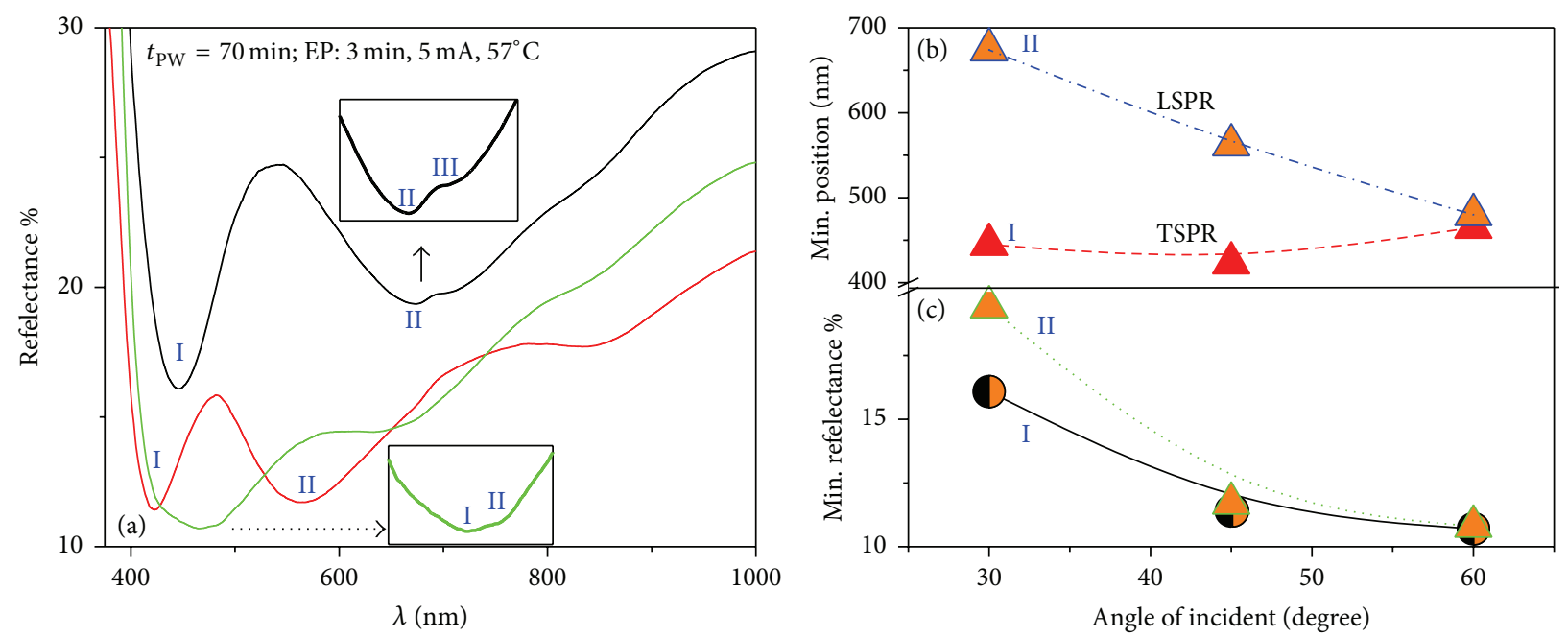

$$
\begin{aligned}
\theta & =30^{\circ} \\
\theta & =45^{\circ} \\
\theta & =60^{\circ}
\end{aligned}
$$
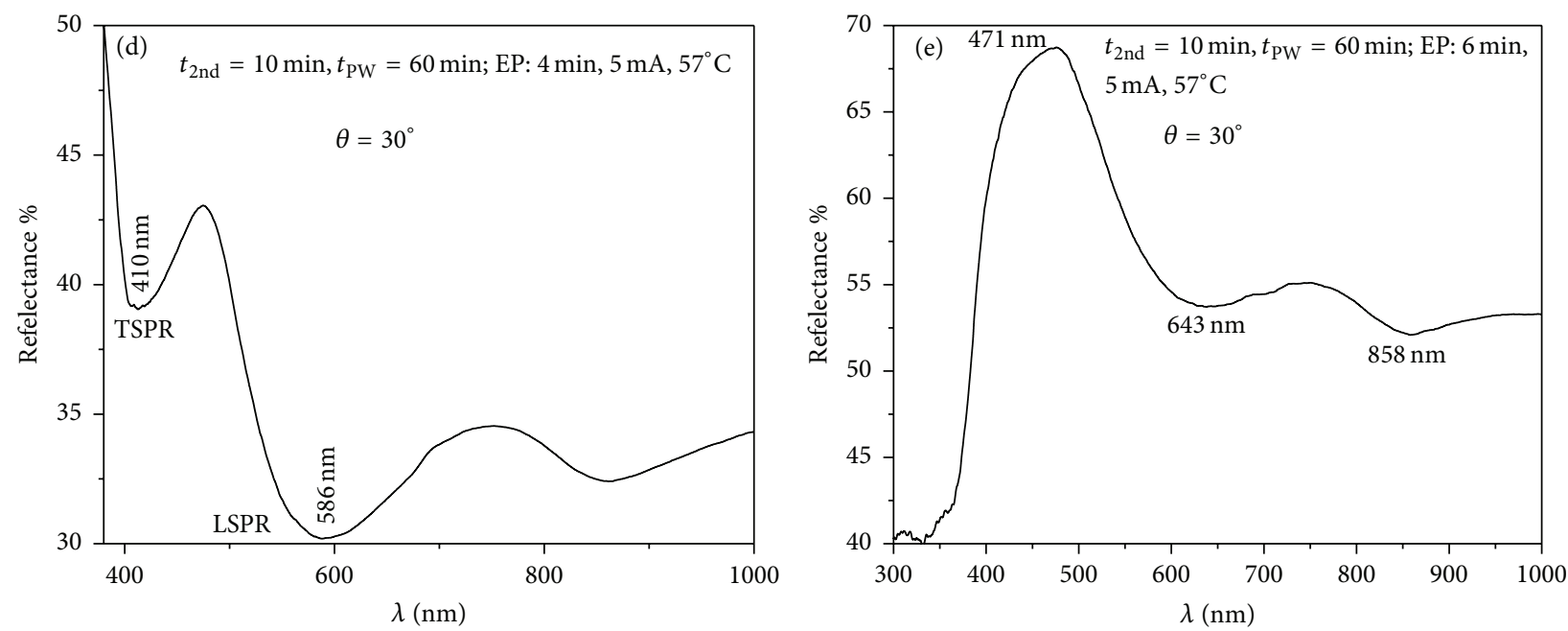

Figure 4: Reflectance spectra of (a) PAA membrane pore widened for $70 \mathrm{~min}$ and electrodeposited with Au at $5 \mathrm{~mA}$ and $57^{\circ} \mathrm{C}$ for $3 \mathrm{~min}$ and measured at different angles of incident and $(b, c)$ their spectroscopic analysis and PAA membranes pore widened for 60 min and electrodeposited at the same conditions for (d) $4 \mathrm{~min}$ and (e) $6 \mathrm{~min}$.

behavior for peak II. This may be attributed and ascribed to the existence of this band very close to the emission band of PAA as a result of the mixed emission from $\mathrm{F}$ and $\mathrm{F}^{+}$ centers. The value of the calculated band gap energy for this membrane was found to be $3.06 \pm 0.02 \mathrm{eV}$.

To compare the effect of the pore widening time on the optical reflectance of PAA membranes, Figure 3(g) shows the optical reflectance spectra of two PAA membranes pore widened for 60 and $70 \mathrm{~min}$. The average reflectivity and the peak intensity are decreased and the bandwidth increased as the pore widening time increased. The peak position exponentially red-shifts as the PW time decreased, Figure 3(h), and the peak reflectance $\%$ nonlinearly decreases, Figure $3(\mathrm{~h})$. The refractive indices are calculated using (1) to be $1.61 \pm 0.20$ at $\mathrm{PW}=60 \mathrm{~min}$ and $1.36 \pm 0.26$ at $\mathrm{PW}=70 \mathrm{~min}$.
Figure 4(a) shows the reflection spectra of PAA membrane pore widened for $70 \mathrm{~min}$ and electrodeposited with $\mathrm{Au}$ at $5 \mathrm{~mA}$ and $57^{\circ} \mathrm{C}$ for $3 \mathrm{~min}$ for incident angles of 30 , 45 , and 60 . The transverse surface plasmon resonance (TSPR $[6,19]$, minimum I) appeared for all angles between 423 and $465 \mathrm{~nm}$. Its position was slightly red-shifted and dependent on the incident angle as shown in Figure 4(b). On the other hand, the longitudinal surface plasmon resonance (LSPR [6, 19,23 ], minimum II) was strongly blue-shifted and dependent on the angle of incidence. It shifts from 674 to $480 \mathrm{~nm}$ as the angle increased from $30^{\circ}$ to $60^{\circ}$. At $60^{\circ}$, LSPR and TSPR are very close to each other, and the two minima are coalesced to a broad minimum as shown in the lower inset of Figure 4(a). It could be seen from Figure 4(c) that the intensity (reflectance $\%$ at the minima) of the surface plasmon 
resonances decreased nonlinearly with the angle of incidence. For large angles of incidence, the electric field oscillations of the light wave have a small component parallel to the rode axis and thus interact with the LSP and large component parallel to the rod diameter and thus interact with the TSP. From this data, it is clear that this composite is optically anisotropic with a uniaxial symmetry.

Figures 4(d) and 4(e) show the reflectance spectra measured at an angle of $30^{\circ}$ and obtained from $\mathrm{Au}$ nanorod nanoarrays of cylindrical shape and pillar shape, respectively. For the cylindrical nanorod arrays, Figure 4(d), the TSPR and LSPR are located at 410 and $586 \mathrm{~nm}$, respectively. Both values are blue-shifted compared to those of Au/PAA composite in Figure 4(a), which may be ascribed to several reasons. Firstly, the length of the nanorods in this sample is greater than the height of the PAA pores, and then the change of dielectric environment from $\mathrm{Al}_{2} \mathrm{O}_{3}$ to air at the top surface of this nanorod arrays may lead to that blue shift [6]. Secondly, the nanorods in this array are very close, and then the interrod coupling strength increases and may result in strong blue shift [32]. For the Au nanopillar array, Figure 4(e), strong and wide reflection peak is centered at $471 \mathrm{~nm}$ with FWHM of $200 \mathrm{~nm}$. This may imply the blue shift of TSPR to $\lambda \leq$ $350 \mathrm{~nm}$. Also, there are only two weak minima centered at 643 and $858 \mathrm{~nm}$, which may be related to LSPRs. The strong enhancement of the plasmon field occurs at contacting surfaces between Au nanorods and leads to superfocusing of electromagnetic waves. Then, the reflected waves of PAA are strongly modulated by hexagonal arrays of Au nanorods.

\section{Conclusion}

In conclusion, we have successfully electrodeposited $\mathrm{Au}$ nanorods arrays inside the pores of PAA membranes of different diameters. The morphologies, structures, and optical properties of as-prepared, posttreated, and $\mathrm{Au}$-coated PAA membranes were studied by FE-SEM and UV-Vis spectrophotometer. The effect of combined (cathodic polarization and pore widening) method and electrodeposition time on the PAA and Au/PAA morphologies and optical properties under the illumination of the unpolarized light incident at different angles were addressed. By increasing the pore widening time, the regularity of the pores increased, the number of defects or cracks decreased, and homogeneity of the hexagonal arrangement increased. By increasing the deposition time from $4 \mathrm{~min}$ to $6 \mathrm{~min}$, the morphology of the nanorods is tuned from cylindrical shape to pillar shape. By increasing the angle of incidence, the contrast of the interference fringes is decreased, the peaks are linearly shifted to longer wavelengths, and the separation between the peaks is increased. Using the modified Kubelka-Munk radiative transfer model, the energy gap of PAA is increased from 2.83 to $3.06 \mathrm{eV}$ after $70 \mathrm{~min}$ pore widening. Also, the refractive index is decreased from 1.84 to 1.36 as the pore widening time increased to $70 \mathrm{~min}$. The TSPR is red-shifted from 423 to $465 \mathrm{~nm}$ and the LSPR is blue-shifted from 674 to $480 \mathrm{~nm}$ as the angle increased from $30^{\circ}$ to $60^{\circ}$. The SPRs are strongly affected by the rod morphology. The advantages of the fabrication approach and the enhanced and controlled optical properties make these samples suitable for nanophotonic and nanoelectronics devices.

\section{Competing Interests}

The author declares that there is no conflict of interests regarding the publication of this paper.

\section{Acknowledgments}

This work was partially supported by the Science and Technology Development Fund (STDF) program of Egypt (Grant no. 4617).

\section{References}

[1] D. Losic and A. Santos, Electrochemically Engineered Nanoporous Materials: Methods, Properties and Applications, Springer Series in Materials Science, Springer, Zug, Switzerland, 2015.

[2] C. W. Lai, "Surface morphology and growth of anodic titania nanotubes films: photoelectrochemical water splitting studies," Journal of Nanomaterials, vol. 2015, Article ID 820764, 7 pages, 2015.

[3] M. Shaban, A. M. Ahmed, E. M. Abdel-Rahman, and H. Hamdy, "Fabrication and characterization of micro/nanoporous $\mathrm{Cr}$ film for sensing applications," Microporous and Mesoporous Materials, vol. 198, pp. 115-121, 2014.

[4] M. Shaban, "Effect of pore thickness and the state of polarization on the optical properties of hexagonal nanoarray of $\mathrm{Au} /$ nanoporous anodic alumina membrane," Journal of Nanomaterials, vol. 2015, Article ID 347486, 9 pages, 2015.

[5] X. Y. Han and W. Z. Shen, "Improved two-step anodization technique for ordered porous anodic aluminum membranes," Journal of Electroanalytical Chemistry, vol. 655, no. 1, pp. 56-64, 2011.

[6] M. Shaban, H. Hamdy, F. Shahin, and S.-W. Ryu, "Strong surface plasmon resonance of ordered gold nanorod array fabricated in porous anodic alumina template," Journal of Nanoscience and Nanotechnology, vol. 10, no. 5, pp. 3034-3037, 2010.

[7] M. R. Kim, D. K. Lee, and D.-J. Jang, “Template-based electrochemically controlled growth of segmented multimetal nanorods," Journal of Nanomaterials, vol. 2010, Article ID 203756, 7 pages, 2010.

[8] C. Y. Han, Z.-L. Xiao, H. H. Wang, X.-M. Lin, S. Trasobares, and R. E. Cook, "Facile synthesis of highly aligned multiwalled carbon nanotubes from polymer precursors," Journal of Nanomaterials, vol. 2009, Article ID 562376, 11 pages, 2009.

[9] M. Shaban, M. Ali, K. Abdelhady, and H. Hamdy, " $\mathrm{Al}_{2} \mathrm{O}_{3}$ and $\mathrm{Sn} / \mathrm{Al}_{2} \mathrm{O}_{3}$ nanowires: fabrication and characterization," Micro \& Nano Letters, vol. 10, no. 7, p. 324, 2015.

[10] G. Wang, C. Shi, N. Zhao, and X. Du, "Synthesis and characterization of Ag nanoparticles assembled in ordered array pores of porous anodic alumina by chemical deposition," Materials Letters, vol. 61, no. 18, pp. 3795-3797, 2007.

[11] D. Sung, S. Hong, Y.-H. Kim et al., "Ab initio study of the effect of water adsorption on the carbon nanotube field-effect transistor," Applied Physics Letters, vol. 89, no. 24, Article ID 243110, 2006.

[12] M. Serry, A. Gamal, M. Shaban, and A. Sharaf, "High sensitivity optochemical and electrochemical metal ion sensor," Micro and Nano Letters, vol. 8, no. 11, pp. 775-778, 2013. 
[13] J. Wang, C.-W. Wang, Y. Li, and W.-M. Liu, "Optical constants of anodic aluminum oxide films formed in oxalic acid solution," Thin Solid Films, vol. 516, no. 21, pp. 7689-7694, 2008.

[14] Y. Du, W. L. Cai, C. M. Mo, J. Chen, L. D. Zhang, and X. G. Zhu, "Preparation and photoluminescence of alumina membranes with ordered pore arrays," Applied Physics Letters, vol. 74, article 2951, 1999.

[15] W. L. Xu, H. Chen, M. J. Zheng, G. Q. Ding, and W. Z. Shen, "Optical transmission spectra of ordered porous alumina membranes with different thicknesses and porosities," Optical Materials, vol. 28, no. 10, pp. 1160-1165, 2006.

[16] Y. Yamamoto, N. Baba, and S. Tajima, "Coloured materials and photoluminescence centres in anodic film on aluminium," Nature, vol. 289, no. 5798, pp. 572-574, 1981.

[17] X. H. Wang, T. Akahane, H. Orikasa, T. Kyotani, and Y. Y. $\mathrm{Fu}$, "Brilliant and tunable color of carbon-coated thin anodic aluminum oxide films," Applied Physics Letters, vol. 91, no. 1, Article ID 011908, 2007.

[18] R. Nikov, N. Nedyalkov, P. A. Atanasov, M. Terakawa, H. Shimizu, and M. Obara, "Tuning the optical properties of gold nanostructures fabricated on flexible substrates," Applied Surface Science, vol. 264, pp. 779-782, 2013.

[19] M. Shaban, H. Hamdy, F. Shahin, and S.-W. Ryu, "Optical properties of porous anodic alumina membrane uniformly decorated with ultra-thin porous gold nanoparticles arrays," Journal of Nanoscience and Nanotechnology, vol. 11, no. 2, pp. 941-952, 2011.

[20] H. Li, C. Kan, Z. Yi, X. Ding, Y. Cao, and J. Zhu, "Synthesis of one dimensional gold nanostructures," Journal of Nanomaterials, vol. 2010, Article ID 962718, 8 pages, 2010.

[21] X. Zhang, B. Sun, H. Guo, N. Tetreault, H. Glessen, and R. H. Friend, "Large-area two-dimensional photonic crystals of metallic nanocylinders based on colloidal gold nanoparticles," Applied Physics Letters, vol. 90, Article ID 133114, 2007.

[22] M. Shaban, A. G. A. Hady, and M. Serry, "A new sensor for heavy metals detection in aqueous media," IEEE Sensors Journal, vol. 14, no. 2, pp. 436-441, 2014.

[23] X. Lang, L. Qian, P. Guan, J. Zi, and M. Chen, "Localized surface plasmon resonance of nanoporous gold," Applied Physics Letters, vol. 98, no. 9, Article ID 093701, 2011.

[24] L. H. Qian, X. Q. Yan, T. Fujita, A. Inoue, and M. W. Chen, "Surface enhanced Raman scattering of nanoporous gold: smaller pore sizes stronger enhancements," Applied Physics Letters, vol. 90, no. 15, Article ID 153120, 2007.

[25] M. Shaban, H. Hamdy, F. Shahin, and S.-W. Ryu, "Fabrication of ordered Cr nanostructures by self agglomeration on porous anodic alumina membranes," Journal of Nanoscience and Nanotechnology, vol. 11, no. 8, pp. 7145-7150, 2011.

[26] M. Shaban, M. Ali, K. Abdel-Hady, and H. Hamdy, "Selfagglomeration of tin nanoparticle array on porous anodic alumina membranes: fabrication and characterization," Current Nanoscience, vol. 11, no. 2, pp. 214-221, 2015.

[27] G. Meng, A. Cao, J. Cheng et al., "Ordered Ni nanowire tip arrays sticking out of the anodic aluminum oxide template," Journal of Applied Physics, vol. 97, no. 6, Article ID 064303, 2005.

[28] M. Shaban, H. Hamdy, F. Shahin, J. Park, and S.-W. Ryu, "Uniform and reproducible barrier layer removal of porous anodic alumina membrane," Journal of Nanoscience and Nanotechnology, vol. 10, no. 5, pp. 3380-3384, 2010.

[29] L. P. Johnson and J. G. Matisons, "Synthesis of high aspectratio gold nanowires with highly porous morphology," ISRN Nanomaterials, vol. 2012, Article ID 502960, 9 pages, 2012.
[30] M. E. Nasir, B. Hamilton, and D. W. E. Allsop, "Optical measurements of nanoporous anodic alumina formed on $\mathrm{Si}$ using novel X-ray spectroscopy set up CLASSIX," Nuclear Instruments and Methods in Physics Research, Section B: Beam Interactions with Materials and Atoms, vol. 268, no. 3-4, pp. 251253, 2010.

[31] A. B. Murphy, "Band-gap determination from diffuse reflectance measurements of semiconductor films, and application to photoelectrochemical water-splitting," Solar Energy Materials and Solar Cells, vol. 91, no. 14, pp. 1326-1337, 2007.

[32] P. R. Evans, R. Kullock, W. R. Hendren, R. Atkinson, R. J. Pollard, and L. M. Eng, "Optical transmission properties and electric field distribution of interacting 2D silver nanorod arrays," Advanced Functional Materials, vol. 18, no. 7, pp. 10751079,2008 

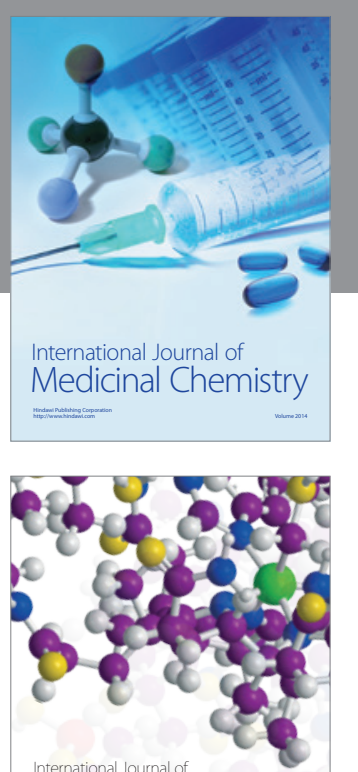

Carbohydrate Chemistry

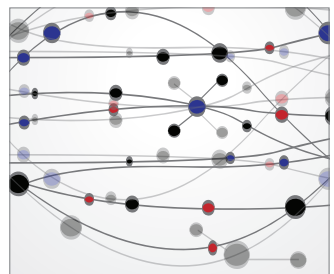

The Scientific World Journal
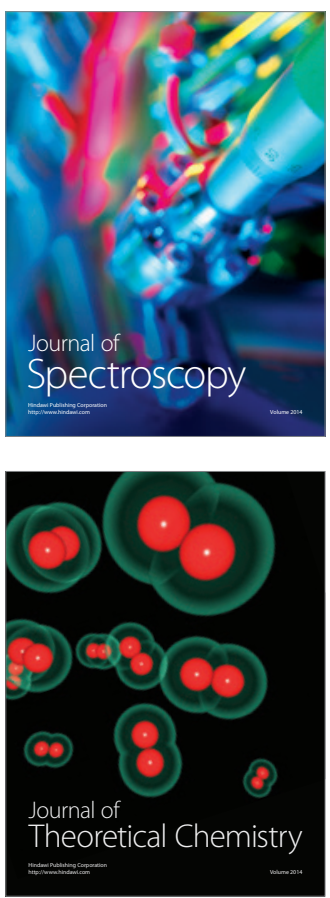
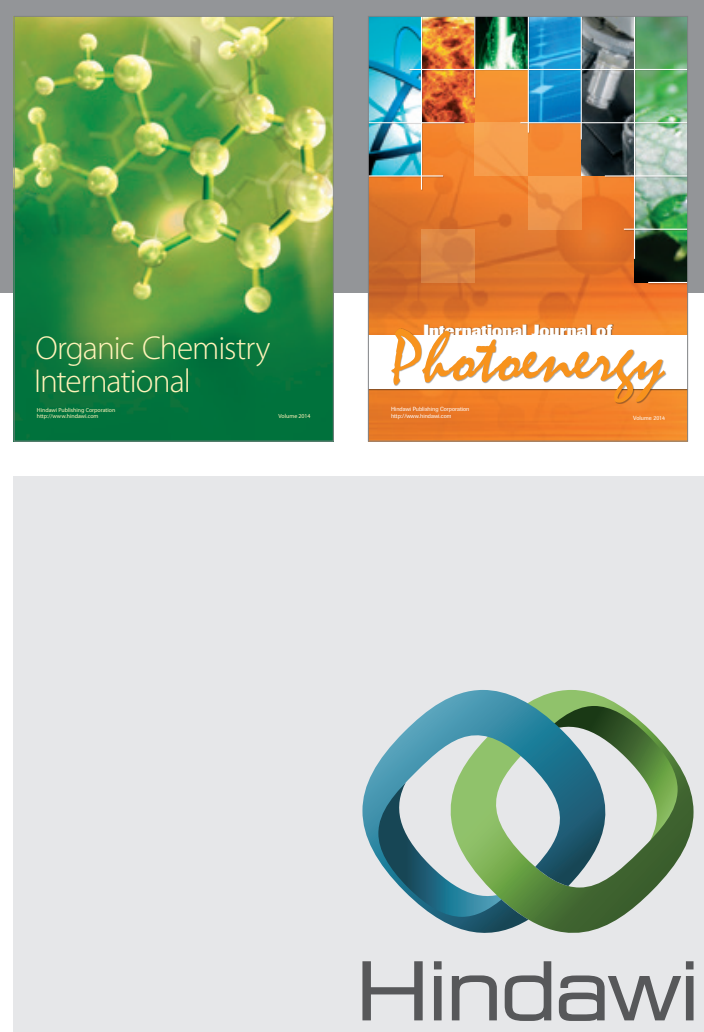

Submit your manuscripts at

http://www.hindawi.com

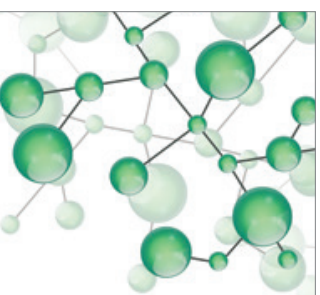

International Journal of

Inorganic Chemistry

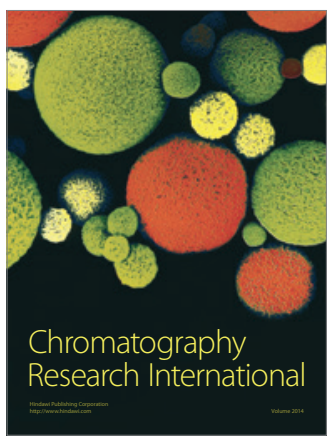

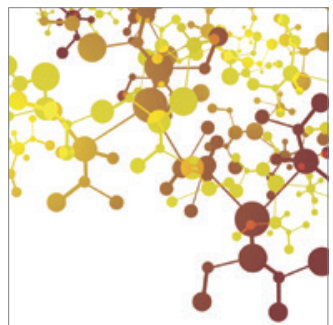

Applied Chemistry
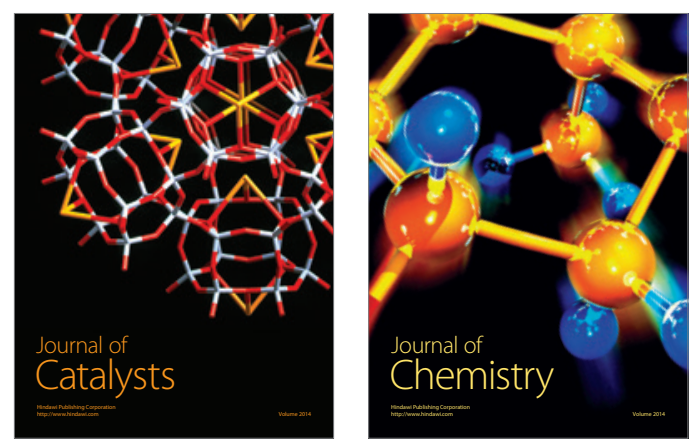
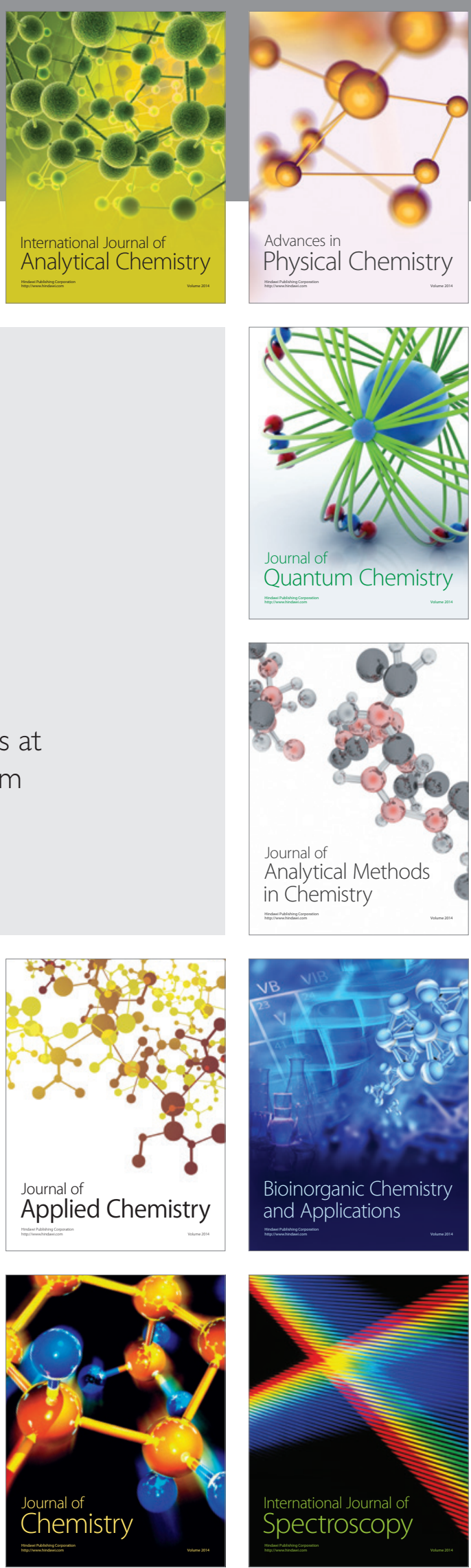